\title{
The Intensity Correlation Function in Evanescent Wave Scattering
}

\author{
B. Cichocki ${ }^{1}$, E. Wajnryb ${ }^{2}$, J. Bławzdziewicz ${ }^{3}$, J.K.G. Dhont ${ }^{4}$, P. Lang ${ }^{4}$ \\ (1) Institute of Theoretical Physics \\ University of Warsaw \\ Hoża 69, 00-681 Warsaw \\ Poland \\ (2) Institute of Fundamental Technological Research \\ Polish Academy of Sciences \\ ul. Pawińskiego 5B, 02-106 Warsaw \\ Poland \\ (3) Department of Mechanical Engineering and Department of Physics \\ Yale University \\ P.O. Box 208286 New Haven, CT 06520-8286 \\ USA. \\ (4) Research Center Jülich \\ IFF-7, Weiche Materie \\ D-52425 Jülich \\ Germany.
}

\begin{abstract}
As a first step towards the interpretation of dynamic light scattering with evanescent illumination, in order to probe dynamics near walls, we develop a theory for the initial slope of the intensity auto-correlation function for suspensions of interacting spheres. An expression for the first cumulant is derived that is valid for arbitrary concentrations, which generalizes a well-known expression for the short-time, wave-vector dependent collective diffusion coefficient in bulk to the case where a wall is present. Explicit expressions and numerical results for the various contributions to the initial slope are obtained within a leading order virial expansion. The dependence of the initial slope on the components of the wave vector parallel and perpendicular to the wall, as well as the dependence on the evanescent-light penetration depth are discussed. For the hydrodynamic interactions
\end{abstract}


between colloids and between the wall, which are essential for a correct description of the near-interface dynamics, we include both far-field and lubrication contributions. Lubrication contributions are essential to capture the dynamics as probed in experiments with small penetration depths. Simulations have been performed to verify the theory, and to estimate the extent of the concentration range where the virial expansion is valid. The computer algorithm developed for this purpose will also be of future importance for the interpretation of experiments, and to develop an understanding of near-interface dynamics, at high colloid concentrations.

\section{Introduction}

The understanding of interactions of macromolecules with a solid/liquid interface is important for many applications of synthetic systems as well as biological processes. Interactions of colloids with a solid wall can lead to structure formation at the wall. The static interactions between colloidal particles and interfaces that underly such near-interface structure formation are relatively well understood within the framework of established potentials, such as the DLVO potential resulting from a combination of charge- and van der Waals-interactions, the depletion potential resulting from polymer depletion in the region between the colloid and the interface, and steric repulsion between chemically grafted polymer coatings. Besides structural order, also the dynamics of colloids is affected by the presence of an interface. Contrary to the near-interface equilibrium structures, the dynamics is determined not only through the above mentioned static interactions, but also through hydrodynamic interactions. These interactions are mediated via the solvent, and include interactions of the colloids with the interface and colloid-colloid interactions. As yet, little is known about near-interface dynamics of concentrated suspensions.

The dynamics of sub-micron sized colloids can be probed by evanescent wave dynamic light scattering (EWDLS), where the intensity auto-correlation function of light that is scattered from an evanescent wave is measured. The penetration depth of the evanescent wave can be tuned by the angle of the incident laser beam with respect to the interface. For small penetration depths, the dynamics of colloids very close to the wall is probed, whereas for larger penetration depths also particles which are further away from the wall contribute to the measured intensity correlation function. Contrary to bulk dynamic light scattering, a theory that is necessary for the interpretation of EWDLS-correlation functions is not yet existing. In a very early attempt [1], the EWDLS-correlation function for very dilute dispersions has been calculated with the neglect of hydrodynamic interactions with the wall. Since, however, hydrodynamic interactions are essential, this theory has limited value for the interpretation of experimental data.

As a first step towards the interpretation of EWDLS-correlations functions, the initial slope of the correlation function for very dilute dispersions of spherical colloids has been expressed in terms of single-particle hydrodynamic friction functions [2, 3]. Numerical values for the initial slope can be obtained, using the semi-analytic predictions for the hydrodynamic functions in the presence of a wall that have been derived in the sixties by Brenner and coworkers [4, 5], 
and which have been verified experimentally $[3,6,7,8,9,10]$. The theoretically predicted dependence of the initial slope on the penetration depth and the components of the scattering wave vector parallel and perpendicular to the wall are in accordance with experiments [3]. These experiments have become possible only recently, by means of EWDLS-equipment that allows to vary the components of the scattering wave vector parallel and perpendicular to the wall independently $[2,11]$.

The capability to vary the parallel and perpendicular components of the scattering wave vector independently is a necessary requirement for the study of the anisotropic diffusion of colloids near a wall. To our knowledge, the only experimental study on diffusion of colloidal spheres at high concentration is Ref.[12]. In this paper, EWDLS data for PMMA particles close to a wall are discussed for a wide range of volume fractions. Part of the interpretation of the data relies on the assumption that the expression for the initial slope of the EWDLScorrelation function has the same form as for very dilute systems $[2,3]$, where the parallel and perpendicular "diffusion coefficients" are now concentration dependent. For particle volume fractions above $\phi \geq 0.35$, the observed near wall diffusion coefficients superimpose with the corresponding bulk diffusion coefficients of the particles. That is, at high concentrations, the hydrodynamic effect of the wall is small. This indicates that hydrodynamic interactions with the wall are partly screened.

It is the purpose of this paper to develop a theory for the initial slope of the EWDLScorrelation function for suspensions of interacting spheres. An exact expression is derived for the initial slope, for which a leading order virial expansion is derived. Numerical results are obtained from an additional analysis of the hydrodynamic interactions between two colloidal spheres and the wall. The calculation of hydrodynamic mobilities, including lubrication contributions, is based on the HYDROMULTIPOLE code implemented according to Ref.[13]. The multidimensional integrals in the above procedure have been performed numerically. Additional Monte-Carlo computer simulations are performed that show that the virial expansion for the initial slope of the EWDLS-correlation function is valid up to quite high concentrations.

This paper is organized as follows. After an introduction, a general expression for the initial slope of the EWDLS-correlation function for interacting spheres is derived in section 2. This generalizes the well-known and much studied expression for the short-time collective diffusion coefficient in bulk to the case where a wall is present. In section3, the various contributions to the initial slope are expressed in terms of ensemble averages, which are tractable for numerical evaluation. The virial expansions of the various contributions are discussed in section 4 . The details of the computer simulations for arbitrary concentrations are given in section 5 and numerical results are discussed in section 6 . Section 7 contains a summary, concluding remarks and suggestions for further work. 


\section{Short-time Evanescent Wave Dynamics}

In this section we derive an expression for the short-time decay of light scattering correlation functions as measured by the evanescent-wave scattering from suspensions of mono-disperse spherical colloids. This expression is a generalization of a well-known result for bulk light scattering to evanescent wave illumination. For infinite penetration depths, the results for evanescent wave scattering of course reduce to the well-known expressions for scattering from bulk samples. In case of the standard dynamic light scattering from bulk samples, the initial decay of the electric field auto-correlation function $\hat{g}_{1}$ is exponential in time,

$$
g_{1} \equiv<E_{s}(t) E_{s}^{\star}(t=0)>\sim \exp \left\{-\Gamma_{1} t\right\},
$$

where $E_{s}$ is the instantaneous scattered electric field strength, and $\Gamma_{1}$ is the first cumulant, which is equal to [14],

$$
\Gamma_{1}=q^{2} D_{0} \frac{H(q)}{S(q)},
$$

where $q$ is the scattering vector, $D_{0}$ is the diffusion coefficient of a free diffusing sphere, $H$ is the "hydrodynamic function" and $S$ is the static structure factor. The hydrodynamic function is related to hydrodynamic interaction mobilities $\boldsymbol{\mu}_{i j}$ as,

$$
H(q)=\frac{1}{\mu_{0} N} \sum_{i, j}^{N}<\hat{\mathbf{q}} \cdot \boldsymbol{\mu}_{i j} \cdot \hat{\mathbf{q}} \exp \left\{i \mathbf{q} \cdot\left(\mathbf{r}_{i}-\mathbf{r}_{j}\right)\right\}>,
$$

where $N$ is the total number of particles in the volume that is probed in a light scattering experiment, $\hat{\mathbf{q}}$ is the unit vector in the direction of $\mathbf{q}$, the brackets $\langle\cdots\rangle$ refer to ensemble averaging, and $\mathbf{r}_{k}$ is the position of the center of sphere $k$. The hydrodynamic mobility connects the velocities of spheres to the hydrodynamic forces acting on them,

$$
\mathbf{U}_{i}=\boldsymbol{\mu}_{i j} \cdot \mathbf{F}_{j}
$$

where $\mathbf{U}_{i}$ is the velocity of particle $i$ due to the force $\mathbf{F}_{j}$ applied to particle $j$. Here, $\mu_{0}=$ $1 / 6 \pi \eta_{0} a$ is the mobility of a non-interacting sphere, which is connected to the Einstein diffusion coefficient $D_{0}$ as $k_{B} T \mu_{0}$ (where $\eta_{0}$ is the solvent viscosity, $a$ is the radius of the sphere, $k_{B}$ is Boltzmann's constant and $T$ the temperature). Furthermore, the static structure factor is defined as,

$$
S(q)=\frac{1}{N} \sum_{i, j}^{N}<\exp \left\{i \mathbf{q} \cdot\left(\mathbf{r}_{i}-\mathbf{r}_{j}\right)\right\}>,
$$

which can in principle be obtained independently from static light scattering experiments.

There are a number of theories devoted to the prediction of the concentration and wavevector dependence of $H(q)$. These theories have been verified by experiments on various colloidal systems with different pair-interaction potentials [15, 16, 17, 18]. 
There are two fundamental differences between standard dynamic light scattering and evanescent-wave scattering. First of all, the illumination intensity varies on a length scale comparable to the size of the colloidal particles. When the wall is located at $z=0$, the incident intensity varies like $\sim \exp \{-\kappa z\}$, where $\kappa^{-1}$ is the intensity-penetration depth. The instantaneous scattered electric field strength $E_{s}$ is now given by,

$$
E_{s} \sim \sum_{j} \exp \left\{-\frac{1}{2} \kappa z_{j}\right\} \exp \left\{i \mathbf{q} \cdot \mathbf{r}_{j}\right\}
$$

where it is assumed that the particles are dielectrically isotropic. Secondly, the hydrodynamic mobilities $\boldsymbol{\mu}_{i j}$ are affected by the presence of the wall. Two particles near a wall interact hydrodynamically in a different way as when the particles are far away from the wall, since shear waves generated by one of the particles are affected by the wall before reaching the other particle. Hereafter, we shall denote the hydrodynamic mobility in the presence of a wall by $\boldsymbol{\mu}_{i j}^{w}$, where the superscript " $w$ " stands for "wall".

The scattered electric field is a Gaussian variable with zero mean, so that Fick's theorem can be applied to express the intensity auto-correlation function in terms of ensemble averages of bilinear products of the scattered electric field strength. Furthermore, the average $<E_{s}(t) E_{s}(t=$ $0)>$ is equal to 0 , provided that there is translational invariance in directions parallel to the wall. This implies that the Siegert relation also holds in case of evanescent-wave scattering $[2],[3]$,

$$
<i_{s}(t) i_{s}(t=0)>=I^{2}+\left|g_{1}\right|^{2},
$$

where $i_{s}$ is the instantaneous intensity that is scattered by the colloidal spheres, and $I=<i_{s}>$ is the average intensity. Just like for standard dynamic light scattering on bulk samples, it is thus sufficient to consider the field auto-correlation function. According to eq.(6), this correlation function is given by,

$$
g_{1} \sim \sum_{i, j=1}^{N}<\exp \left\{-\frac{1}{2} \kappa\left(z_{i}(t)-z_{j}(t=0)\right)\right\} \exp \left\{-i \mathbf{q} \cdot\left(\mathbf{r}_{i}(t)-\mathbf{r}_{j}(t=0)\right)\right\}>
$$

The short-time behaviour of this correlation function can be calculated, starting from the general expression for the equilibrium time-correlation function of two phase-functions $f$ and $g$,

$$
<f(t) g(t=0)>=\int d \mathbf{R} f(\mathbf{R}) \exp \{\hat{\mathcal{L}} t\}\left[g(\mathbf{R}) P_{\mathrm{eq}}^{\mathrm{w}}(\mathbf{R})\right]
$$

where $\mathbf{R}=\left\{\mathbf{r}_{1}, \mathbf{r}_{2}, \cdots \mathbf{r}_{N}\right\}$ is the $3 N$-dimensional position in phase space, $P_{\mathrm{eq}}^{\mathrm{w}} \sim \exp \{-\beta \Phi\}$ is the Boltzmann probability density function of $\mathbf{R}$, and $\Phi$ is the total interaction potential of the $N$ interacting spheres, including the interactions with the wall. Furthermore, $\hat{\mathcal{L}}$ is the Smoluchowski operator that describes the temporal evolution of the conditional probability density function (pdf) $P\left(\mathbf{R}, t \mid \mathbf{R}_{0}\right)$ of the position $\mathbf{R}$ of the colloidal sphere at time $t$, given that its position is $\mathbf{R}_{0}$ at time $t=0$,

$$
\frac{\partial P\left(\mathbf{R}, t \mid \mathbf{R}_{0}\right)}{\partial t}=\hat{\mathcal{L}} P\left(\mathbf{R}, t \mid \mathbf{R}_{0}\right)
$$


The Smoluchowski operator is given by (with $h$ an arbitrary phase function),

$$
\hat{\mathcal{L}} h=k_{B} T \sum_{i, j=1}^{N} \nabla_{i} \cdot \boldsymbol{\mu}_{i j}^{w} \cdot\left[\nabla_{j} h+\beta h \nabla_{j} \Phi\right] .
$$

Note that the hydrodynamic mobilities $\boldsymbol{\mu}_{i j}^{w}$ as well as the total potential energy $\Phi$ include both interactions between the colloidal spheres and the wall. Like for the bulk mobilities, the mobilities in the presence of a wall satisfy the Lorentz symmetry relation [19],[20],

$$
\boldsymbol{\mu}_{i j}^{w}=\left[\boldsymbol{\mu}_{j i}^{w}\right]^{T},
$$

where "T" stands for "transpose". Comparing the general expression (8) with our specific function shows that,

$$
\begin{aligned}
& f(\mathbf{R})=\sum_{i=1}^{N} \exp \left\{-\frac{1}{2} \kappa z_{i}\right\} \exp \left\{-i \mathbf{q} \cdot \mathbf{r}_{i}\right\} \\
& g(\mathbf{R})=\sum_{j=1}^{N} \exp \left\{-\frac{1}{2} \kappa z_{j}\right\} \exp \left\{+i \mathbf{q} \cdot \mathbf{r}_{j}\right\}
\end{aligned}
$$

A short-time expansion can now be obtained by approximating the exponential operator to leading order in time,

$$
\exp \{\hat{\mathcal{L}} t\}=\hat{\mathcal{I}}+\hat{\mathcal{L}} t+" \mathcal{O}\left(t^{2}\right) "
$$

where $\hat{\mathcal{I}}$ is the identity operator. Substitution of eqs.(13) and (14) into eq.(9) leads to the short-time expansion of $g_{1}$. This expression is most conveniently evaluated by noting that,

$$
\int d \mathbf{R} f(\mathbf{R}) \hat{\mathcal{L}}\left[P_{\mathrm{eq}}^{\mathrm{w}}(\mathbf{R}) g(\mathbf{R})\right]=-k_{B} T \sum_{i, j=1}^{N} \int d \mathbf{R} P_{\mathrm{eq}}^{\mathrm{w}}(\mathbf{R})\left[\nabla_{i} f(\mathbf{R})\right] \cdot \boldsymbol{\mu}_{i j}^{w} \cdot\left[\nabla_{j} g(\mathbf{R})\right],
$$

where a partial integration has been performed, and it is used that $P_{\mathrm{eq}}^{\mathrm{w}} \sim \exp \{-\beta \Phi\}$. The square brackets $[\cdots]$ are used to indicate that the action of the $\nabla$-operators is limited to the functions within these brackets. Using this expression together with our specific functions in eq.(13), readily leads to the following expression for the first cumulant,

$$
\Gamma_{1}=D_{0}\left[\frac{1}{2} \kappa \hat{\mathbf{e}}_{z}-i \mathbf{q}\right] \cdot \frac{\mathbf{H}_{w}(\kappa, \mathbf{q})}{S_{w}(\kappa, \mathbf{q})} \cdot\left[\frac{1}{2} \kappa \hat{\mathbf{e}}_{z}+i \mathbf{q}\right]
$$

where $\hat{\mathbf{e}}_{z}=(0,0,1)$ is the unit vector normal to the wall pointing into the suspension,

$$
\mathbf{H}_{w}(\kappa, \mathbf{q})=\frac{\kappa}{\mu_{0} n A} \sum_{i, j}^{N}<\exp \left\{-\frac{1}{2} \kappa\left(z_{i}+z_{j}\right)\right\} \boldsymbol{\mu}_{i j}^{w} \exp \left\{i \mathbf{q} \cdot\left(\mathbf{r}_{i}-\mathbf{r}_{j}\right)\right\}>,
$$


is the hydrodynamic function, and,

$$
S_{w}(\kappa, \mathbf{q})=\frac{\kappa}{n A} \sum_{i, j}^{N}<\exp \left\{-\frac{1}{2} \kappa\left(z_{i}+z_{j}\right)\right\} \exp \left\{i \mathbf{q} \cdot\left(\mathbf{r}_{i}-\mathbf{r}_{j}\right)\right\}>
$$

is the wall structure factor. In eqs. $(17,18), n A / \kappa$ is the number of particles within the probed scattering volume, with $n$ the bulk particle number density and $A$ the illuminated area. The brackets $\langle\cdots\rangle$ in eqs. $(17,18)$ denote the average with respect to $P_{\text {eq }}^{\mathrm{w}}$.

The above result (16-18) is a generalization of the bulk expressions (2,3) and (5) for the short-time behaviour of $g_{1}$. For very large penetration depths $(\kappa \rightarrow 0)$, they reduce to these well-known bulk expressions, as they should.

For very dilute suspensions of spheres, we have,

$$
\boldsymbol{\mu}_{i j}^{w}=\delta_{i j}\left[\left(\hat{\mathbf{e}}_{x} \hat{\mathbf{e}}_{x}+\hat{\mathbf{e}}_{y} \hat{\mathbf{e}}_{y}\right) \mu_{\|}^{w}+\hat{\mathbf{e}}_{z} \hat{\mathbf{e}}_{z} \mu_{\perp}^{w}\right],
$$

where $\delta_{n m}$ is the Kronecker delta, where $\mu_{\|}^{w}$ and $\mu_{\perp}^{w}$ are the scalar mobilities for motion parallel and perpendicular to the wall, respectively, and $\hat{\mathbf{e}}_{x}$ and $\hat{\mathbf{e}}_{y}$ are two orthogonal unit vectors parallel to the wall. Furthermore, in case of hard-core interactions between the colloidal sphere and the wall, we have $\Phi=0$ for $z<a$ (with $a$ the radius of a colloidal sphere), and $\Phi=$ constant for $z>a$. The first cumulant in eq.(16) is then easily shown to reduce to,

$$
\Gamma_{1}=\left(\frac{1}{4} \kappa^{2}+q_{\perp}^{2}\right)<D_{\perp}>+q_{\|}^{2}<D_{\|}>
$$

where $q_{\perp}$ and $q_{\|}$are the components of the scattering wave vector $\mathbf{q}$ perpendicular and parallel to the wall, respectively, and where,

$$
<D_{\perp, \|}>=k_{B} T \kappa \int_{z>a} d z \exp \{-\kappa(z-a)\} \mu_{\perp, \|}^{w}(z) .
$$

This reproduces the expression that was derived in Ref.[3].

An essential difference between dilute and interacting systems is, that there are contributions to $\Gamma_{1}$ which do not vary with $\sim q_{\perp}^{2}$, . Due to invariant properties of the system it immediately follows from eq.(16) that the first cumulant can be written as,

$$
\Gamma_{1}=\frac{D_{0}}{S_{w}}\left[\left(\frac{1}{4} \kappa^{2}+q_{\perp}^{2}\right) H_{\perp, \perp}+q_{\|}^{2} H_{\|,\|}+\frac{1}{2} i q_{\|} \kappa\left(H_{\perp, \|}-H_{\|, \perp}\right)+q_{\|} q_{\perp}\left(H_{\perp, \|}+H_{\|, \perp}\right)\right]
$$

with,

$$
\begin{aligned}
H_{\perp, \perp}\left(\kappa, q_{\perp}, q_{\|}\right) & =\hat{\mathbf{e}}_{z} \cdot \mathbf{H}_{w}(\kappa, \mathbf{q}) \cdot \hat{\mathbf{e}}_{z}, \\
H_{\|,\|}\left(\kappa, q_{\perp}, q_{\|}\right) & =\hat{\mathbf{q}}_{\|} \cdot \mathbf{H}_{w}(\kappa, \mathbf{q}) \cdot \hat{\mathbf{q}}_{\|}, \\
H_{\perp, \|}\left(\kappa, q_{\perp}, q_{\|}\right) & =\hat{\mathbf{e}}_{z} \cdot \mathbf{H}_{w}(\kappa, \mathbf{q}) \cdot \hat{\mathbf{q}}_{\|}, \\
H_{\|, \perp}\left(\kappa, q_{\perp}, q_{\|}\right) & =\hat{\mathbf{q}}_{\|} \cdot \mathbf{H}_{w}(\kappa, \mathbf{q}) \cdot \hat{\mathbf{e}}_{z},
\end{aligned}
$$


and where we decomposed $\mathbf{q}$ into horizontal and vertical parts,

$$
\mathbf{q}=\mathbf{q}_{\|}+q_{\perp} \hat{\mathbf{e}}_{z}=q_{\|} \hat{\mathbf{q}}_{\|}+q_{\perp} \hat{\mathbf{e}}_{z}
$$

It follows from the symmetry relation in eq.(12) that $H_{\perp, \|}=H_{\|, \perp}^{\star}$, so that eq.(22) can also be written as,

$$
\Gamma_{1}=\frac{D_{0}}{S_{w}}\left[\left(\frac{1}{4} \kappa^{2}+q_{\perp}^{2}\right) H_{\perp}+q_{\|}^{2} H_{\|}+\frac{1}{2} q_{\|} \kappa H_{I}+q_{\|} q_{\perp} H_{R}\right]
$$

where for brevity we defined,

$$
\begin{aligned}
H_{\perp}\left(\kappa, q_{\perp}, q_{\|}\right) & =H_{\perp, \perp}\left(\kappa, q_{\perp}, q_{\|}\right) \\
H_{\|}\left(\kappa, q_{\perp}, q_{\|}\right) & =H_{\|,\|}\left(\kappa, q_{\perp}, q_{\|}\right) \\
H_{I}\left(\kappa, q_{\perp}, q_{\|}\right) & =2 \Im H_{\|, \perp}\left(\kappa, q_{\perp}, q_{\|}\right), \\
H_{R}\left(\kappa, q_{\perp}, q_{\|}\right) & =2 \Re H_{\|, \perp}\left(\kappa, q_{\perp}, q_{\|}\right)
\end{aligned}
$$

where $\Im$ and $\Re$ stand for "imaginary" and "real part of", respectively. In our previous notation for dilute systems, we denoted $D_{0} H_{\perp, \|}$ as $\left\langle D_{\perp, \|}\right\rangle$ (see eqs. $(16,20,21)$ ). Note that $H_{I, R}$ are both equal to 0 for very dilute systems. As will be seen later, for concentrated systems, both $H_{R}$ and $H_{I}$ vanish when $q_{\perp}$ is fixed and $q_{\|} \rightarrow 0$, but in case $q_{\|}$is fixed and $q_{\perp} \rightarrow 0$ only the component $H_{R}$ vanishes. The "off-diagonal contributions" $H_{I, R}$ are generally significant, and cannot be neglected against the "diagonal contributions" $H_{\perp, \|}$. The off-diagonal contributions also become zero for very large $q$. The diagonal contributions can be related to self-diffusive properties for such large wave vectors, as will be seen later.

\section{The Wall Structure Factor and Hydrodynamic Tensor}

In this section we introduce the basic functions and operators that are used to express the wall hydrodynamic tensor(17) and the wall structure factor (18) in a form that is tractable for explicit, numerical evaluation. Furthermore, we will define response operators appropriate for the present problem, and generalize the notion of self-diffusion to the situation where a wall is present.

In order to proceed, we first introduce the $s$-particle distribution functions $g\left(\mathbf{r}_{1}, \ldots, \mathbf{r}_{s}\right)$, which are given by,

$$
n^{s} g\left(\mathbf{r}_{1}, \ldots, \mathbf{r}_{s}\right)=\lim _{\infty} \frac{N !}{(N-s) !} \int d \mathbf{r}_{s+1} \ldots \int d \mathbf{r}_{n} P_{\mathrm{eq}}^{\mathrm{w}}(\mathbf{R}), \quad s=1,2, \ldots,
$$

and $P_{\mathrm{eq}}^{\mathrm{w}}$ is, as before, the equilibrium distribution function for the system of $N$ particles in a presence of wall. Here, $\lim _{\infty}$ denotes the thermodynamic limit, which is also understood to be taken in the definitions in eqs. $(17,18)$ for the hydrodynamic function and wall structure factor, respectively. In order to carry out the thermodynamic limit, $\lim _{\infty}$, we select the cuboidal 
shape of the system with volume $V=L_{x} L_{y} L_{z}$ and go with $L_{x}, L_{y}$ and $L_{z}$ uniformly to infinity keeping the bulk density $n$ constant. From below the system is bounded by a hard wall at $z=0$, with a surface area $A=L_{x} L_{y}$. Considering the symmetry of the system, the 1-particle distribution function depends only on the distance from the wall, i.e. $g(\mathbf{r})=g(z)$. Due to the same symmetry, for the 2-particle distribution function we have,

$$
g\left(\mathbf{r}, \mathbf{r}^{\prime}\right)=g\left(z, z^{\prime}, \rho\right)
$$

where the symbol $\rho$ denotes the length of the vector $\boldsymbol{\rho}$, which is defined as,

$$
\mathbf{r}-\mathbf{r}^{\prime}=\boldsymbol{\rho}+\left(z-z^{\prime}\right) \mathbf{e}_{z} .
$$

Employing the one and two particle distribution functions, the wall structure factor (18) can be written as,

$$
\begin{aligned}
S_{\mathrm{w}}(\kappa, \mathbf{q}) & =\kappa \int_{0}^{\infty} d z e^{-\kappa z} g(z)+ \\
& 2 \pi n \kappa \int_{0}^{\infty} d z \int_{0}^{\infty} d z^{\prime} e^{-\kappa\left(z+z^{\prime}\right) / 2} \cos \left[q_{\perp}\left(z-z^{\prime}\right)\right] \int_{0}^{\infty} d \rho \rho h\left(z, z^{\prime}, \rho\right) \mathrm{J}_{0}\left(q_{\|} \rho\right),
\end{aligned}
$$

where,

$$
h\left(z, z^{\prime}, \rho\right)=g\left(z, z^{\prime}, \rho\right)-g(z) g\left(z^{\prime}\right) .
$$

In addition, the integration over the angle $\varphi$, defined by the relation $\hat{\mathbf{q}}_{\|} \cdot \hat{\boldsymbol{\rho}}=\cos \varphi$, has been carried out. The Bessel function $\mathrm{J}_{0}$ in eq.(30) enters due to the relation [21]

$$
\int_{0}^{2 \pi} e^{i q \rho \cos \varphi} d \varphi=2 \pi \mathrm{J}_{0}(q \rho) .
$$

In the limit of large penetrations depths, where $\kappa=0$, the eq.(30) for the wall structure factor reduces to,

$$
S(q)=1+4 \pi n \int_{0}^{\infty} d r r^{2} h(r) \frac{\sin \{q r\}}{q r},
$$

where $h$ is then total bulk correlation function. This corresponds to the expression for the structure factor in eq.(5)

The tensor $\mathbf{H}_{\mathrm{w}}\left(\kappa, q_{\perp}, q_{\|}\right)$is similarly given in terms of one and two-particle-integrals as,

$$
\begin{aligned}
\mathbf{H}_{\mathrm{w}}\left(\kappa, q_{\perp}, q_{\|}\right)= & \kappa \int_{0}^{\infty} d z e^{-\kappa z} \mathbf{A}(z)+ \\
& n \kappa \int_{0}^{\infty} d z \int_{0}^{\infty} d z^{\prime} e^{-\kappa \frac{z+z^{\prime}}{2}} e^{i q_{\perp}\left(z-z^{\prime}\right)} \int d \boldsymbol{\rho} e^{i \mathbf{q}_{\|} \cdot \boldsymbol{\rho}} \mathbf{B}\left(z, z^{\prime}, \boldsymbol{\rho}\right),
\end{aligned}
$$

where the kernel operators $\mathbf{A}$ and $\mathbf{B}$ are defined as,

$$
\mathbf{A}(z)=\frac{1}{n \mu_{0}} \lim _{\infty}\left\langle\sum_{i=1}^{N} \delta\left(\mathbf{r}-\mathbf{r}_{i}\right) \boldsymbol{\mu}_{i i}^{\mathrm{w}}(\mathbf{R})\right\rangle
$$




$$
\mathbf{B}\left(z, z^{\prime}, \boldsymbol{\rho}\right)=\frac{1}{n^{2} \mu_{0}} \lim _{\infty}\left\langle\sum_{i \neq j}^{N} \delta\left(\mathbf{r}-\mathbf{r}_{i}\right) \delta\left(\mathbf{r}^{\prime}-\mathbf{r}_{j}\right) \boldsymbol{\mu}_{i j}^{\mathrm{w}}(\mathbf{R})\right\rangle .
$$

Due to the invariant properties, the tensors $\mathbf{A}$ and $\mathbf{B}$ have the following representations,

$$
\mathbf{A}(z)=A_{\perp}(z) \hat{\mathbf{e}}_{z} \hat{\mathbf{e}}_{z}+A_{\|}(z) \mathbf{1}_{\|}
$$

and,

$$
\begin{aligned}
\mathbf{B}\left(z, z^{\prime}, \boldsymbol{\rho}\right)= & B_{a}\left(z, z^{\prime}, \rho\right) \hat{\mathbf{e}}_{z} \hat{\mathbf{e}}_{z}+B_{b}\left(z, z^{\prime}, \rho\right)\left(\mathbf{1}_{\|}-\hat{\boldsymbol{\rho}} \hat{\boldsymbol{\rho}}\right)+ \\
& B_{c}\left(z, z^{\prime}, \rho\right) \hat{\boldsymbol{\rho}} \hat{\boldsymbol{\rho}}+B_{d}\left(z, z^{\prime}, \rho\right) \hat{\boldsymbol{\rho}} \hat{\mathbf{e}}_{z}+B_{e}\left(z, z^{\prime}, \rho\right) \hat{\mathbf{e}}_{z} \hat{\boldsymbol{\rho}},
\end{aligned}
$$

where,

$$
\mathbf{1}_{\|}=\mathbf{1}-\hat{\mathbf{e}}_{z} \hat{\mathbf{e}}_{z} .
$$

Since the tensor B obeys the Lorentz symmetry (see eq.(12)),

$$
\mathbf{B}\left(z^{\prime}, z,-\boldsymbol{\rho}\right)=\mathbf{B}^{\mathrm{T}}\left(z, z^{\prime}, \boldsymbol{\rho}\right),
$$

its elements have the following properties,

$$
\begin{aligned}
& B_{\alpha}\left(z^{\prime}, z, \rho\right)=B_{\alpha}\left(z, z^{\prime}, \rho\right), \quad \alpha=a, b, c \\
& B_{d}\left(z^{\prime}, z, \rho\right)=-B_{e}\left(z, z^{\prime}, \rho\right) .
\end{aligned}
$$

We can thus write the hydrodynamic factors (26) as,

$$
\begin{gathered}
H_{\perp}\left(\kappa, q_{\perp}, q_{\|}\right)=\kappa \int_{0}^{\infty} d z e^{-\kappa z} A_{\perp}(z)+ \\
2 \pi n \kappa \int_{0}^{\infty} d z \int_{0}^{\infty} d z^{\prime} e^{-\kappa\left(z+z^{\prime}\right) / 2} \cos \left[q_{\perp}\left(z-z^{\prime}\right)\right] \times \\
\int_{0}^{\infty} d \rho \rho B_{a}\left(z, z^{\prime}, \rho\right) \mathrm{J}_{0}\left(q_{\|} \rho\right), \\
H_{\|}\left(\kappa, q_{\perp}, q_{\|}\right)=\kappa \int_{0}^{\infty} d z e^{-\kappa z} A_{\|}(z)+ \\
\pi n \kappa \int_{0}^{\infty} d z \int_{0}^{\infty} d z^{\prime} e^{-\kappa\left(z+z^{\prime}\right) / 2} \cos \left[q_{\perp}\left(z-z^{\prime}\right)\right] \int_{0}^{\infty} d \rho \rho \\
{\left[B_{b}\left(z, z^{\prime}, \rho\right)\left(\mathrm{J}_{0}\left(q_{\|} \rho\right)+\mathrm{J}_{2}\left(q_{\|} \rho\right)\right)+B_{c}\left(z, z^{\prime}, \rho\right)\left(\mathrm{J}_{0}\left(q_{\|} \rho\right)-\mathrm{J}_{2}\left(q_{\|} \rho\right)\right)\right],(43)} \\
H_{\mathrm{R}}\left(\kappa, q_{\perp}, q_{\|}\right)=-4 \pi n \kappa \int_{0}^{\infty} d z \int_{0}^{\infty} d z^{\prime} e^{-\kappa\left(z+z^{\prime}\right) / 2} \sin \left[q_{\perp}\left(z-z^{\prime}\right)\right] \int_{0}^{\infty} d \rho \rho B_{d}\left(z, z^{\prime}, \rho\right) \mathrm{J}_{1}\left(q_{\|} \rho\right)
\end{gathered}
$$


and,

$$
H_{\mathrm{I}}\left(\kappa, q_{\perp}, q_{\|}\right)=4 \pi n \kappa \int_{0}^{\infty} d z \int_{0}^{\infty} d z^{\prime} e^{-\kappa\left(z+z^{\prime}\right) / 2} \cos \left[q_{\perp}\left(z-z^{\prime}\right)\right] \int_{0}^{\infty} d \rho \rho B_{d}\left(z, z^{\prime}, \rho\right) \mathrm{J}_{1}\left(q_{\|} \rho\right) .
$$

To arrive at the above expressions, the following recurrence relation has been used for the Bessel function $J_{n}[21]$,

$$
J_{n}^{\prime}(x)=\frac{1}{2}\left[\mathrm{~J}_{n-1}(x)-\mathrm{J}_{n+1}(x)\right] .
$$

In the limit $\kappa \rightarrow 0$ the first cumulant (25) with the $H$-components (42-45) reduces to the cumulant in eq.(2) for bulk samples.

Just like for the bulk hydrodynamic function in eq.(3), the corresponding wall-hydrodynamic function can be written as a sum of a self- and distinct-part. The self part is the $q$-independent contribution containing the operator $\mathbf{A}$, and the $q$-dependent distinct part involves the operator B. The self part is the only remaining contribution for large wave vectors. Contrary to the static structure factor (5) in bulk, there is a non-trivial self part to the wall structure factor $S_{\mathrm{w}}(\kappa, \mathbf{q})$, which is equal to,

$$
S_{\mathrm{w}}^{\text {self }}(\kappa)=\kappa \int_{0}^{\infty} d z e^{-\kappa z} g(z) .
$$

In bulk, the self part of the structure factor is simply equal to unity.

In analogy with bulk response functions Ref.[22], we define response functions that relate forces with currents in the presence of a wall. These response functions can be expressed in terms of the above defined operators $\mathbf{A}$ and $\mathbf{B}$. Namely, consider a force field $\mathbf{F}(\mathbf{r})$, where $\mathbf{F}\left(\mathbf{r}_{i}\right)$ is the force acting on a particle located at $\mathbf{r}_{i}$. Following the reasoning in Ref.[22], the resulting mean particle current $\langle\mathbf{j}(\mathbf{r})\rangle$ can be written as,

$$
\langle\mathbf{j}(\mathbf{r})\rangle=\int d \mathbf{r}^{\prime} \mathbf{Y}\left(\mathbf{r}, \mathbf{r}^{\prime}\right) \cdot \mathbf{F}\left(\mathbf{r}^{\prime}\right)
$$

where the response function $\mathbf{Y}$, for the case of a suspension in the presence of a wall, is given by,

$$
\mathbf{Y}\left(\mathbf{r}, \mathbf{r}^{\prime}\right)=n \mu_{0} \delta\left(\mathbf{r}-\mathbf{r}^{\prime}\right) \mathbf{A}(z)+n^{2} \mu_{0} \mathbf{B}\left(z, z^{\prime}, \boldsymbol{\rho}\right) .
$$

In addition, the operator $\mathbf{A}$ is related to the self-diffusion matrix $\mathbf{D}_{s}(z)$. The latter is defined by the initial slope of the mean square displacement of a particle, say particle 1, located at a height $z$ at $t=0$,

$$
D_{s, \alpha \beta}(z)=\frac{1}{2} \frac{d}{d t}\left\langle\Delta x_{1 \alpha}(t) \Delta x_{1 \beta}(t)\right\rangle_{\mid t=0},
$$

where the indices $\alpha, \beta=1,2,3$ describe Cartesian components, and $\Delta x_{1 \alpha}(t)$ is the displacement of particle 1 in the direction $\alpha$ during the time $t$. It can be shown from the Smoluchowski equation $(10,11)$ that,

$$
g(z) \mathbf{D}_{s}(z)=D_{0} \mathbf{A}(z) .
$$


Since the matrix $\mathbf{D}_{s}(z)$ has the same invariant property as $\mathbf{A}(z)$ (see eq.(37)), one can introduce the vertical self-diffusion coefficients $D_{s, \perp}$ and the parallel one $D_{s, \|}$ by,

$$
\mathbf{D}_{s}(z)=D_{s, \perp}(z) \hat{\mathbf{e}}_{z} \hat{\mathbf{e}}_{z}+D_{s, \|}(z) \mathbf{1}_{\|} \cdot
$$

In the limit $q_{\|} \rightarrow+\infty$ or $q_{\perp} \rightarrow+\infty$, only the self parts of $\mathbf{H}_{\mathrm{w}}$ and $S_{\mathrm{w}}$ survive. Thus, for sufficiently large $q_{\|}$or $q_{\perp}$, the first cumulant may be approximated by,

$$
\Gamma_{1} \approx q_{\|}^{2}<D_{s, \|}>_{\kappa}+\left(\frac{1}{4} \kappa^{2}+q_{\perp}^{2}\right)<D_{s, \perp}>_{\kappa},
$$

where,

$$
<D_{s, \perp, \|}>_{\kappa}=\frac{\int_{0}^{\infty} d z e^{-\kappa z} g(z) D_{s, \perp, \|}(z)}{\int_{0}^{\infty} d z e^{-\kappa z} g(z)} .
$$

Note that for a dilute system, where interactions between the colloids can be neglected, this reduces to the formula (21). Like for bulk systems, there is no distinction between self diffusion and collective diffusion at infinite dilution.

When $q_{\perp}$ is fixed and $q_{\|} \rightarrow 0$, both $H_{R}$ and $H_{I}$ vanish. One can show this directly from the the Eqs.(44) and (45) or on bases of invariant properties of the system. However, if $q_{\|}$is fixed and $q_{\perp} \rightarrow 0$, only the component $H_{R}$ vanishes.

\section{Virial Expansion}

For moderately concentrated systems we can perform calculations of the wall structure factor $S_{w}$ and components of the wall-hydrodynamic tensor $\mathbf{H}_{w}$ by expanding them in terms of powers of bulk-particle concentration $n$ far from the wall. To this end we expand the distribution functions in eq.(27) as,

$$
g\left(\mathbf{r}_{1}, \ldots, \mathbf{r}_{s}\right)=g^{(0)}\left(\mathbf{r}_{1}, \ldots, \mathbf{r}_{s}\right)+n g^{(1)}\left(\mathbf{r}_{1}, \ldots, \mathbf{r}_{s}\right)+\ldots . \quad s=1,2, \ldots .
$$

By substituting eq.(55) with $s=1$ and 2 into (30) one easily obtains the form of the virial expansion for the factor $S_{w}$,

$$
S_{\mathrm{w}}(\kappa, \mathbf{q})=\sum_{s=1}^{\infty} n^{s-1} S_{\mathrm{w}}^{(s)}(\kappa, \mathbf{q})
$$

where the two first terms are given by,

$$
S_{\mathrm{w}}^{(1)}(\kappa, \mathbf{q})=\kappa \int_{0}^{\infty} d z e^{-\kappa z} g^{(0)}(z)
$$

and,

$$
\begin{aligned}
S_{\mathrm{w}}^{(2)}(\kappa, \mathbf{q}) & =\kappa \int_{0}^{\infty} d z e^{-\kappa z} g^{(1)}(z)+ \\
& 2 \pi \kappa \int_{0}^{\infty} d z \int_{0}^{\infty} d z^{\prime} e^{-\kappa\left(z+z^{\prime}\right) / 2} \cos \left[q_{\perp}\left(z-z^{\prime}\right)\right] \int_{0}^{\infty} d \rho \rho h^{(0)}\left(z, z^{\prime}, \rho\right) \mathrm{J}_{0}\left(q_{\|} \rho\right),
\end{aligned}
$$


with,

$$
h^{(0)}\left(z, z^{\prime}, \rho\right)=g^{(0)}\left(z, z^{\prime}, \rho\right)-g^{(0)}(z) g^{(0)}(z) .
$$

For a similar expansion of the hydrodynamic tensor we must first introduce the cluster decomposition of mobility tensors,

$$
\begin{array}{r}
\boldsymbol{\mu}_{11}^{\mathrm{w}}(\mathbf{R})=\mu_{0}\left[\mathbf{m}_{11}^{(1)}\left(\mathbf{r}_{1}\right)+\sum_{i \neq 1} \mathbf{m}_{11}^{(2)}\left(\mathbf{r}_{1}, \mathbf{r}_{i}\right)+\sum_{1<i<j} \mathbf{m}_{11}^{(3)}\left(\mathbf{r}_{1}, \mathbf{r}_{i}, \mathbf{r}_{j}\right)+\ldots\right] \\
\boldsymbol{\mu}_{12}^{\mathrm{w}}(\mathbf{R})=\mu_{0}\left[\mathbf{m}_{12}^{(2)}\left(\mathbf{r}_{1}, \mathbf{r}_{2}\right)+\sum_{2<i} \mathbf{m}_{12}^{(3)}\left(\mathbf{r}_{1}, \mathbf{r}_{2}, \mathbf{r}_{i}\right)+\sum_{2<i, j} \mathbf{m}_{12}^{(4)}\left(\mathbf{r}_{1}, \mathbf{r}_{2}, \mathbf{r}_{i}, \mathbf{r}_{j}\right)+\ldots\right] .
\end{array}
$$

The cluster components $\mathbf{m}^{(i)}$ describe hydrodynamic interactions within the corresponding cluster of $i$ particles. With the above, the kernels $\mathbf{A}(z)$ and $\mathbf{B}\left(z, z^{\prime}, \boldsymbol{\rho}\right)$ are thus given by the series expansion,

$$
\begin{gathered}
\mathbf{A}(z)=\sum_{s=1}^{\infty} n^{s-1} \frac{1}{(s-1) !} \int d 2 \ldots \int d s g(1, \ldots, s) \mathbf{m}_{11}^{(s)}(1,2, \ldots, s) \\
\mathbf{B}\left(z, z^{\prime}, \boldsymbol{\rho}\right)=\sum_{s=2}^{\infty} n^{s-2} \frac{1}{(s-2) !} \int d 3 \ldots \int d s g(1, \ldots, s) \mathbf{m}_{12}^{(s)}(1,2, \ldots, s) .
\end{gathered}
$$

To make formulas more compact we have employed here the shorthand notation,

$$
i \equiv \mathbf{r}_{i} \quad \text { and } \quad \int d i \ldots \equiv \int d \mathbf{r}_{i} \ldots
$$

By substituting eq.(55) into eqs.(62) and (63), we arrive at the following expansions of the tensors $\mathbf{A}$ and $\mathbf{B}$,

$$
\begin{gathered}
\mathbf{A}(z)=\sum_{s=1}^{\infty} n^{s-1} \mathbf{A}^{(s)}(z), \\
\mathbf{B}\left(z, z^{\prime}, \boldsymbol{\rho}\right)=\sum_{s=2}^{\infty} n^{s-2} \mathbf{B}^{(s)}\left(z, z^{\prime}, \boldsymbol{\rho}\right),
\end{gathered}
$$

which, with the help of eqs.(42)-(45), lead directly to the virial expansions of the hydrodynamical tensor components. These expansions start with terms of the order $n^{0}$ which contain only the "self" part given by,

$$
\mathbf{A}^{(1)}(z)=g^{(0)}(z) \mathbf{m}_{11}^{(1)}(z) .
$$

This is the expression for $\mathbf{A}$ at infinite dilution. The tensor $\mathbf{B}$ is zero at infinite dilution. The first order corrections due to interactions between the colloids are equal to,

$$
\mathbf{A}^{(2)}(z)=g^{(1)}(z) \mathbf{m}_{11}^{(1)}(z)+\int d 2 g^{(0)}(1,2) \mathbf{m}_{11}^{(2)}(1,2)
$$




$$
\mathbf{B}^{(2)}\left(z, z^{\prime}, \boldsymbol{\rho}\right)=g^{(0)}(1,2) \mathbf{m}_{12}^{(2)}\left(z, z^{\prime}, \boldsymbol{\rho}\right) .
$$

In order to obtain numerical results for the first-order corrections, explicit expression for the distribution functions to zeroth and first order (see eq.(27)), and the appropriate hydrodynamic one- and two-particle cluster components (see eqs. $(60,61)$ ) must be found.

From this point on, we will consider hard-core interactions both for the colloid-colloid and the colloid-wall interactions. These are interactions where the potential is zero when there is no overlap of the colloid- and wall-material, and the potential is infinite on overlap. We also assume that the colloidal spheres are perfect spheres and that the wall is smooth, so that hydrodynamic interactions on very close colloid-colloid and colloid-wall approach can be described by lubrication forces.

The small dimensionless parameter in a density expansion is the bulk volume fraction,

$$
\phi=\frac{4 \pi}{3} a^{3} n
$$

instead of the concentration $n$. With Mayer-graph techniques, where the wall is treated as a very large particle, explicit expressions for the zeroth- and first-order distribution functions are obtained [23]. To zeroth order it is found that,

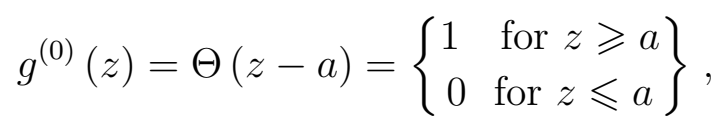

and,

$$
g^{(0)}(1,2)=g^{(0)}\left(z_{1}\right) g^{(0)}\left(z_{2}\right) W(1,2)
$$

where $W(1,2)$ is the characteristic function for non-overlap configurations of two spheres, that is, $W(1,2)=1$ when the distance of the sphere centers $r_{12}$ is larger than $d=2 a$, and 0 otherwise (with $d$ the diameter and $a$ the radius of the spheres). We should recall here that the location of the wall has been chosen as $z=0$ reference level. In the next order, it is found that,

$$
n g^{(1)}(z)=\frac{\phi}{4} \Theta(z-a) \Theta(3 a-z)\left(3-\frac{z}{a}\right)^{2}\left(3+\frac{z}{a}\right) .
$$

The above expressions (71-73) suffice to calculate the two first terms in the virial expansion for the wall structure factor. The integrations in eqs.(57) and for the self part in (58) may be performed analytically. This leads to,

$$
S_{\mathrm{w}}^{(1)}(\kappa)=e^{-\kappa a},
$$

and,

$$
S_{\mathrm{w}}^{\text {self }}{ }^{(2)}(\kappa)=\frac{2 e^{-\kappa a}}{(\kappa d)^{3}}\left[6-3(\kappa d)^{2}+2(\kappa d)^{3}-6 e^{-\kappa d}(1+\kappa d)\right] .
$$

The distinct part in eq.(58) must be done numerically. 
In the case of the wall hydrodynamic tensor we need in addition to eqs.(71-73) the one and two particle cluster components of the mobility matrix. To calculate them, the HYDROMULTIPOLE code implemented according to Ref.[13] has been used. The main idea in this scheme is based on the image representation of a sphere near a hard wall proposed by Cichocki and Jones in Ref.[24]. The latter is a generalization of the result for Stokeslet derived by Blake [25]. In the code the multipole part, which takes care of the longdistance hydrodynamic interactions between spheres is combined with the lubrication corrections accounting for the short-range behavior. The corrections are constructed according to the idea of Durlofsky, Brady and Bossis [26] with the improvements introduced in Ref.[27].

Using the HYDROMULTIPOLE code we generated the one and two particle mobility tensors which appear in the integrands defining the first two virial coefficients for the wall hydrodynamic tensor. Then the one-dimensional integrals over $z$ in these coefficients were evaluated using the Gauss quadrature method. The three-dimensional integrals over $z, z^{\prime}$ and $\rho$ appearing in the definition of the second virial coefficients were evaluated by means of averaging the integrand over a large sequence (typically eight million) of random triplets $z, z^{\prime}, \rho$, where $z$ and $z^{\prime}$ were sampled from the distribution $(\kappa / 2) e^{-\kappa z / 2}$ and $\rho$ was distributed uniformly on the interval of length $30 d$. The remaining part of integral over $\rho$, i.e. from $30 d$ to $\infty$, was carried out analytically using the known asymptotic form of the mobility tensors, which decay as inverse powers of the lateral distance $\rho$. It is worthwhile to mention that the averaging procedure is specific for given intensity-penetration depth, so it had to be repeated for each $\kappa$.

\section{Details of Numerical Simulations}

The initial slope of the EWDLS-correlation functions for arbitrary concentrations is also evaluated by means of Monte Carlo (MC) simulations of an equilibrium ensemble of hydrodynamically coupled spheres in a wall bounded domain. To avoid modeling of an infinite suspension in a half-space bounded by a single wall, our calculations were performed for a system confined by two parallel walls, and periodic conditions were applied in the lateral directions (i.e., the directions parallel to the confining surfaces).

To reduce calculation cost, sample simulations were first performed for channels with different widths. Using these sample calculations, we determined the minimal wall separation for which converged results can be obtained (as discussed below).

Our numerical procedure for the evaluation of the cumulant $\Gamma$ involves the following three main steps: (i) preparation of an equilibrium ensemble of configurations corresponding to a given bulk particle density $n$, (ii) evaluation of the structure factor (18), and (iii) evaluation of the hydrodynamic tensor (17). These three steps are separately discussed below.

Preparation of the equilibrium ensemble - To obtain the equilibrium particle configuration for a given particle number $N$ and specific geometry of the periodic simulation cell, we start from a system with a random particle configuration at a low volume fraction. Then we 
perform a sequence of MC Brownian dynamics equilibration processes, followed by rescaling of the particles to increase the volume fraction without creating particle overlaps. By repeating these steps we obtain a system with a gradually increasing density. When the desired density is achieved, a set of independent equilibrium configurations is obtained via additional Brownian-dynamics process.

Due to formation of a short-ranged layering microstructure in the near-wall regions, the average volume fraction in a two-wall system may significantly differ from the bulk value. Thus, proper adjustment of the average density is required to obtain a matching near-wall particle distribution in systems with different channel widths. To this end we first construct the equilibrium ensemble for a system with a large wall separation $h=h_{0}$ and the required density $n$ in the middle region of the channel. Then we determine the excess particle number per unit area wall $n_{e}$, using the formula

$$
N=A h n+2 A n_{e},
$$

where $N$ is the number of particles in the periodic cell, $A$ is the wall area, and $h=h_{0}$ is the wall separation in the reference system. Having determined the excess surface density of particles in the near-wall regions for a given bulk concentration $n$, the particle number $N=N(h)$ for channels with different width $h$ is obtained from expression (76), with the known values of $n$ and $n_{e}$. Application of this procedure significantly accelerates the convergence of the results to the single-wall limit $h \rightarrow \infty$.

Evaluation of the equilibrium structure factor - For sufficiently large values of the evanescent-wave penetration depth $\kappa^{-1}$ and wave vector $q$, evaluation of the equilibrium structure factor (18) in the wall presence is straightforward. However, to obtain a good numerical convergence (especially at low volume fractions), averaging over a large number of configurations is necessary, because of cancelation of negative and positive contributions. In our simulations we have used $4 \times 10^{4}$ configurations for $\phi=0.10$ and $10^{4}$ configurations for $\phi=0.25$.

Evaluation of the hydrodynamic tensor - The hydrodynamic tensor $\mathbf{H}$ is determined using the periodic version [28] of the Cartesian-representation algorithm [29, 30, 31] for evaluation of the motion of spherical particles in Stokes flow in a parallel-wall channel. Our method combines spherical and Cartesian representations of Stokes flow in the system. The Cartesian representation is used to describe the interaction of the flow with planar walls, and the spherical representation for the flow interaction with the particles. The combined expansion in two sets of basic fields takes into consideration the spherical particle shape and the planar shape of the interface.

To describe interactions between particles with the lateral distance larger than several wall separations $h$, we also use simplifications associated with the quasi-two-dimensional Hele-Shaw character of the far-field flow scattered from the particles [31, 32]. The periodic boundary conditions are implemented by using periodic expressions for the Hele-Shaw far-field flow. 
The numerical results presented in this paper were obtained for the channel width $h=13 d$. By performing simulations at different channel widths we have verified that this wall separation is sufficiently large to yield results with the accuracy within the statistical uncertainty. We find that the convergence of the results is exponential in $h$, because the hydrodynamic field resulting from periodic forcing decays on the length scale $l \sim q_{\|}^{-1}$ away from the wall, and there is an exponential damping factor in eq.(17).

The hydrodynamic tensor $\mathbf{H}$ was determined as an average over $M$ independent $\mathrm{MC}$ trials. To obtain statistical accuracy of the order of $2 \%$, we have used $M$ in the range from $M=30$ for large systems with $N \approx 10^{3}$ particles to $M=400$ for $N \approx 200$ particles.

\section{Results and Discussion}

Numerical results for the two leading order contributions $S_{\mathrm{w}}^{(1)}$ (see eq.(57)) and $S_{\mathrm{w}}^{(2)}$ (see eq.(58)) to the virial expansion for the wall structure factor are shown in Fig.1. In Figs.1a and b, the wall-structure factor contributions are given as functions of $q_{\perp}$ for fixed $q_{\|} d=1.64$, and $q_{\|}$for fixed $q_{\perp} d=2.94$, respectively, both for a penetration depth with $\kappa d=0.96$. These values are typical for experiments that are presently performed. Clearly, the leading-order contribution $S_{\mathrm{w}}^{(1)}$ is independent of the wave vector. Notice, however, that its constant value is not unity, as for the bulk structure factor. This value depends on the penetration depth as is clear from eqs.(57,72). The oscillatory behaviour in Fig.1a is due to layer-structure formation perpendicular to the wall. The oscillatory behaviour is Fig.1b has the same origin as for the bulk structure factor, and is due to ordering that exists around a colloidal sphere due to excluded volume interactions. The dashed horizontal lines in Fig. 1 mark the asymptotic value of $S_{\mathrm{w}}^{(2)}$ for large wave vectors, which, contrary to the bulk structure factor, is different from unity. Again, this value depends on the penetration depth. There is thus a non-trivial dependence of the initial slope of EWDLS-correlation functions on the penetration depth through the wall structure factor.

Using the virial expansions of the hydrodynamic tensors $\mathbf{A}$ and $\mathbf{B}$ together with the virial expansion of the distribution function, as discussed in section 4, leads to the following form for the wall hydrodynamic functions,

$$
H_{\perp}=H_{\perp}^{(1)}+\phi H_{\perp}^{(2)}+\mathcal{O}\left(\phi^{2}\right)
$$

and similarly for $H_{\|}, H_{R}$ and $H_{I}$. The various coefficients $H^{(2)}$ that describe the interaction contributions to the initial slope of the EWDLS-correlation function are given in Figs.2a and b, as a function of $q_{\perp}$ and $q_{\|}$, respectively, for the same fixed values of $q_{\|}, q_{\perp}$ and $\kappa d$ as for the wall structure factor. Like for the bulk hydrodynamic function, the wall-hydrodynamic functions exhibit an oscillatory behaviour as a function of the wave vector, where the peak positions are at about the same wave vectors where the wall-structure factor has its peaks, both for the parallel and the perpendicular wave-vector contributions. The "off-diagonal contribution" $H_{I}$ is seen to be relatively small. The contribution $H_{R}$, however, is of a similar magnitude as the 

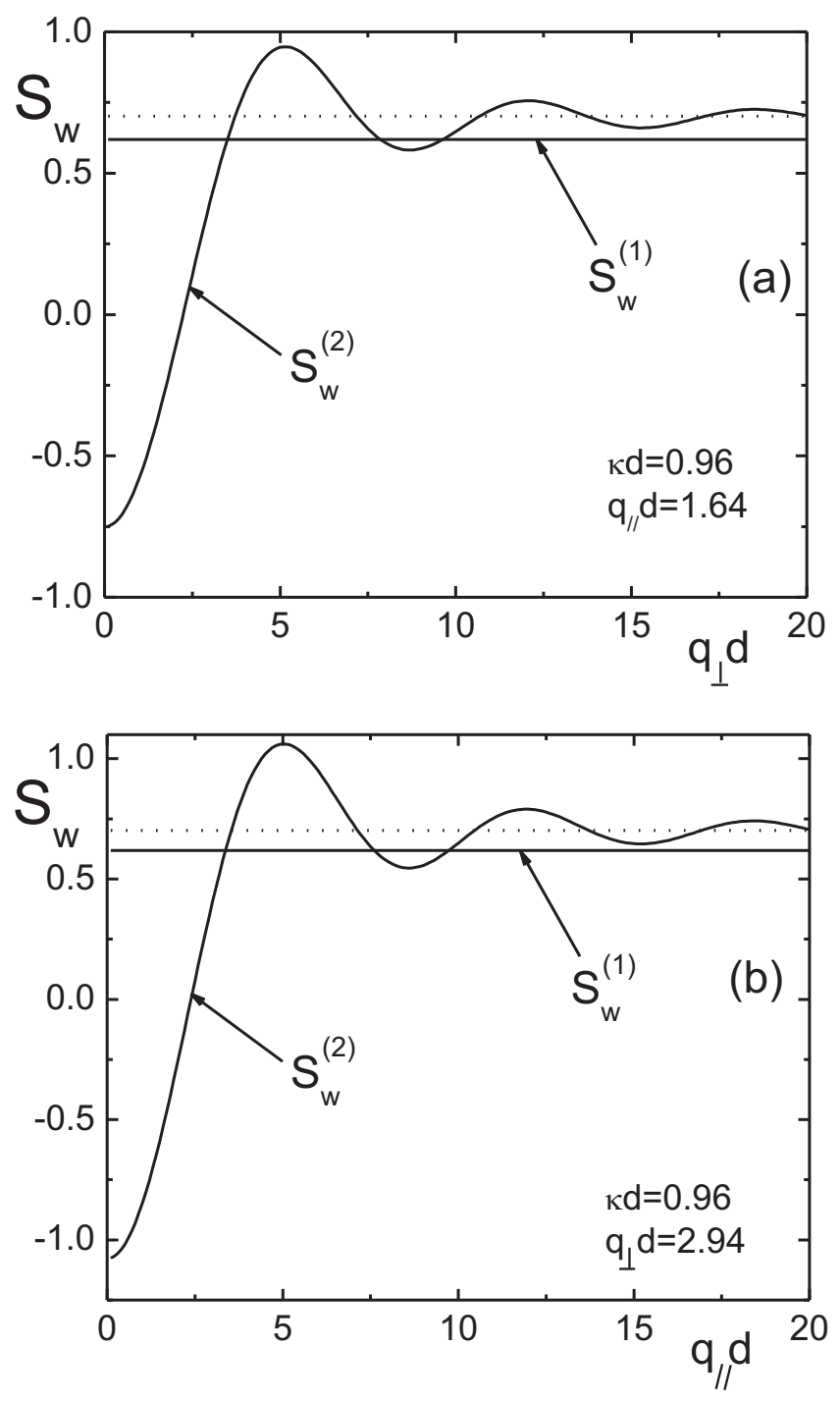

Figure 1: The wall structure factor versus $q_{\perp}$ (a), and $q_{\|}$(b). In (a) $q_{\|} d=1.64$ is fixed, and in (b) $q_{\perp} d=2.94$. In both plots, $\kappa d=0.96$. The functions $S_{w}^{(1)}$ refer to infinite dilution while the functions $S_{w}^{(2)}$ describe the leading order interaction contributions (see eqs. $(57,58)$ ). 

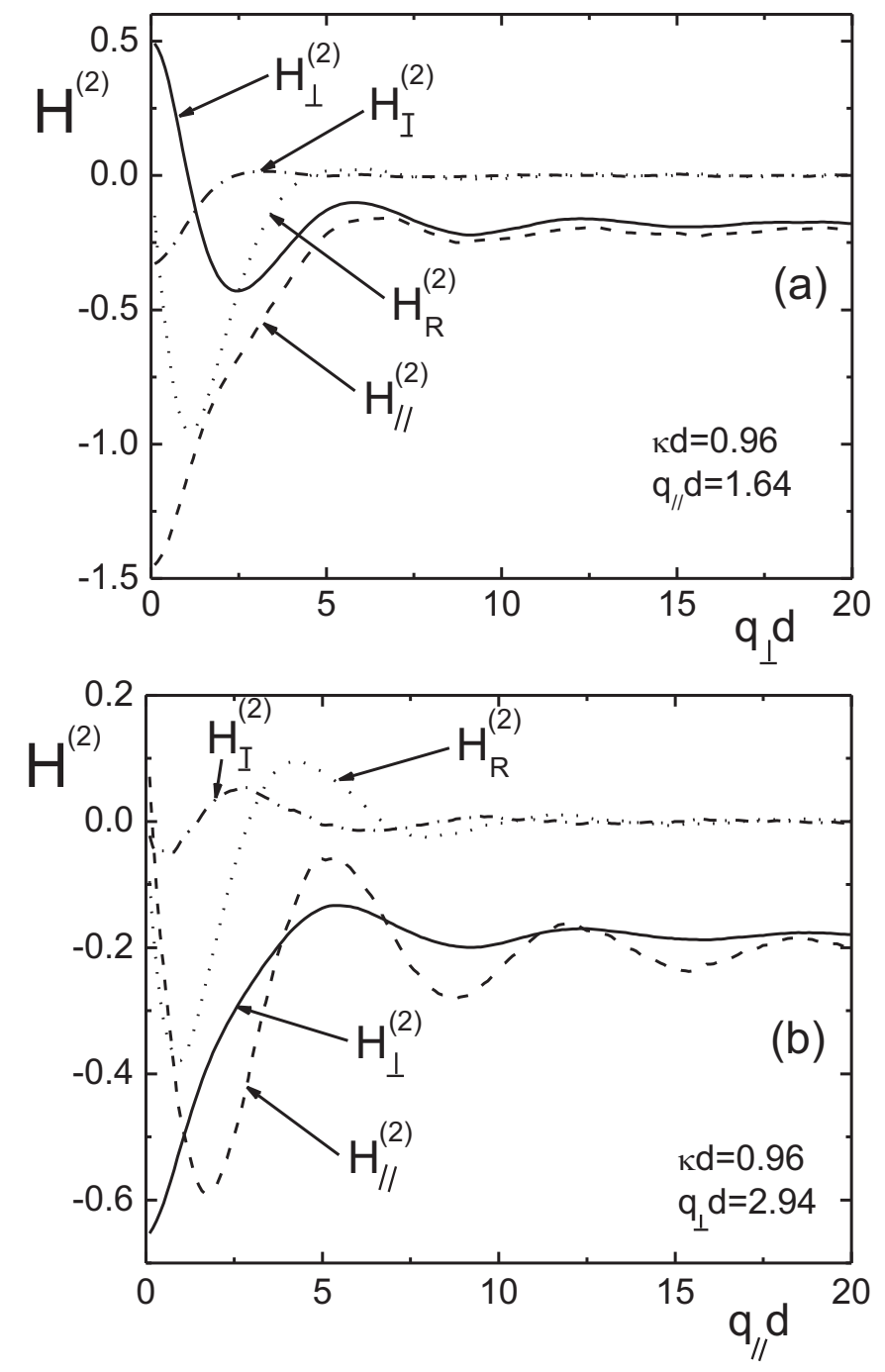

Figure 2: The various components of the hydrodynamic function versus $q_{\perp}$ (a), and $q_{\|}(\mathrm{b})$. In (a) $q_{\|} d=1.64$ is fixed, and in (b) $q_{\perp} d=2.94$. In both plots, $\kappa d=0.96$. The functions $H^{(1)}$ refer to infinite dilution while the functions $H^{(2)}$ describe the leading order interaction contributions (see eq.(77)). 


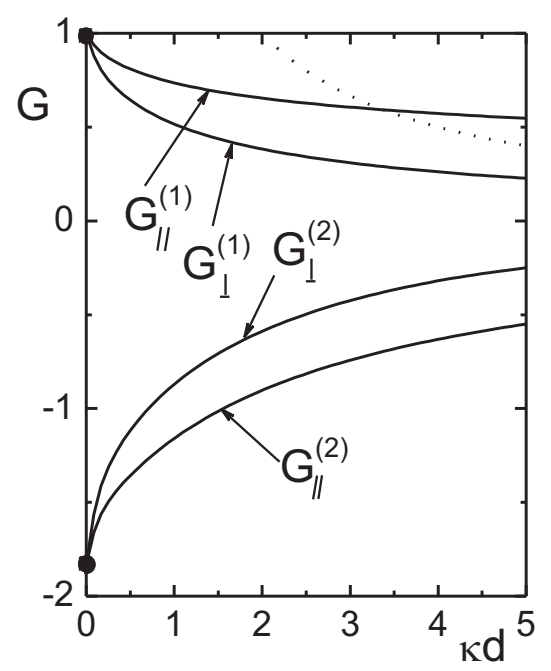

Figure 3: The self-diffusion contributions to the initial slope as defined in eq.(78), as a function of $\kappa d$. The two dots $\bullet$ at $\kappa d=0$ are the limiting values for the infinite dilution contributions $\left(G^{(1)}=1\right)$ and for the leading order interaction contributions $\left.G^{(2)}=-1.831 \cdots\right)$.

"diagonal contributions" $H_{\perp}$ and $H_{\|}$, and will be essential when comparing with experimental results.

Using the virial expansion coefficients $(67,68)$, the vertical- and parallel-self diffusion coefficients can be written as,

$$
\frac{<D_{s, \perp, \|}>_{\kappa}}{D_{0}}=G_{\perp, \|}^{(1)}(\kappa d)+\phi G_{\perp, \|}^{(2)}(\kappa d)+\mathcal{O}\left(\phi^{2}\right)
$$

Numerical results for the virial expansion coefficients as a function of the inverse penetration depth are given in Fig.3. For infinite penetration depths (where $\kappa d=0$ ), the leading coefficients tend to unity, as they should, since for large penetration depths bulk diffusion is probed. The coefficients $G_{\perp, \|}^{(2)}$ must become equal to the coefficient $\alpha=-1.831$ in the well-known virial expansion for the short-time bulk self-diffusion coefficient $D_{s}$ [33],[34],

$$
D_{s}=D_{0}\left[1+\alpha \phi+\mathcal{O}\left(\phi^{2}\right)\right] .
$$

The lower data point $\bullet$ at $\kappa d=0$ corresponds to this limiting value for $\alpha$. The data for both coefficients $G_{\perp, \|}^{(2)}$ indeed seem to converge to this bulk value. At infinite dilution, the self diffusion coefficient in the presence of a wall diminishes for smaller penetration depths due to the hydrodynamic effect of the wall. More interestingly, the concentration dependence of the self-diffusion coefficients weakens for smaller penetration depths. 

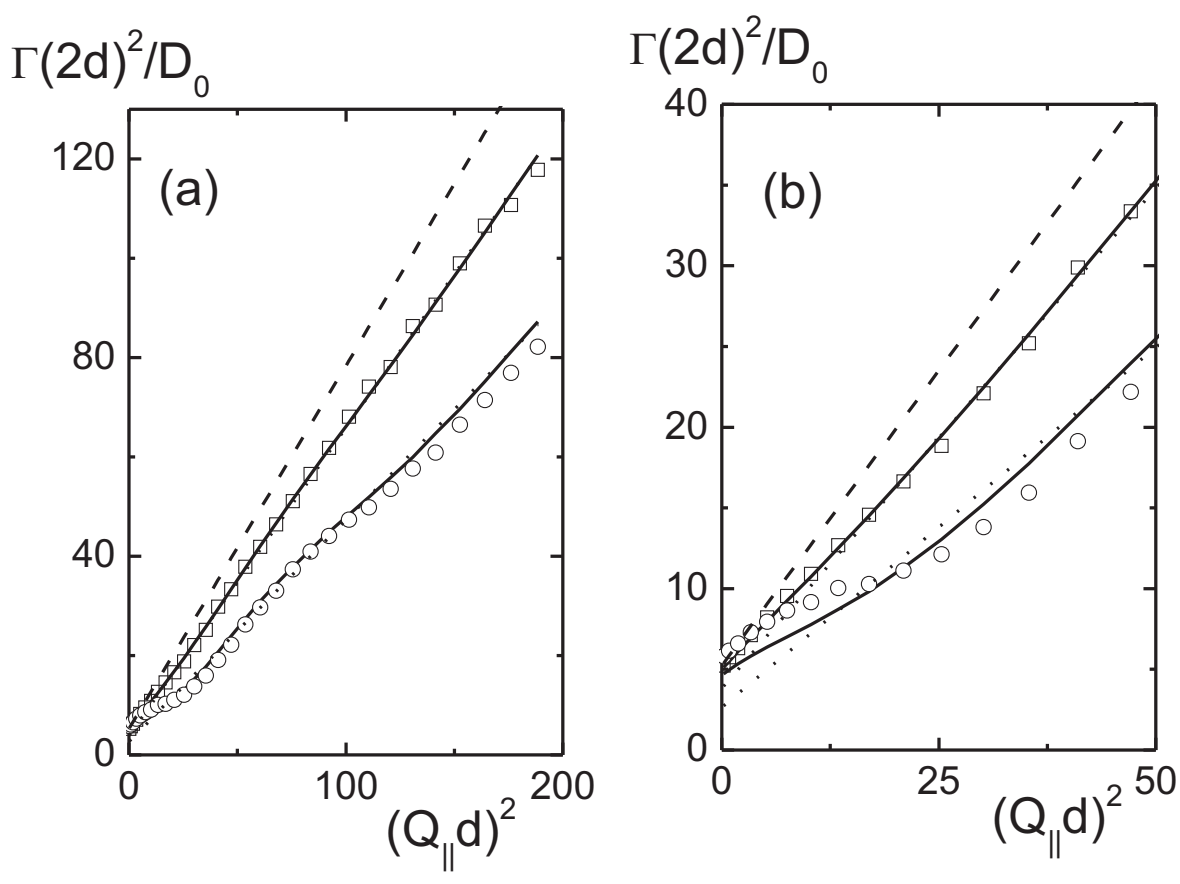

Figure 4: The initial slope as a function of the parallel wave-vector component for $q_{\perp}=2.94$, and $\kappa d=0.96$. Figure (b) is a blow up of figure (a) in order to emphasize the small-wave vector region. The dashed line is for infinite dilution, the middle set of lines is for a volume fraction of $\phi=0.10$ and the lower set of curves for 0.25 . The solid lines are numerical results from the virial expansion, the data points are from simulations, and the dotted lines are the self-diffusion contributions. 

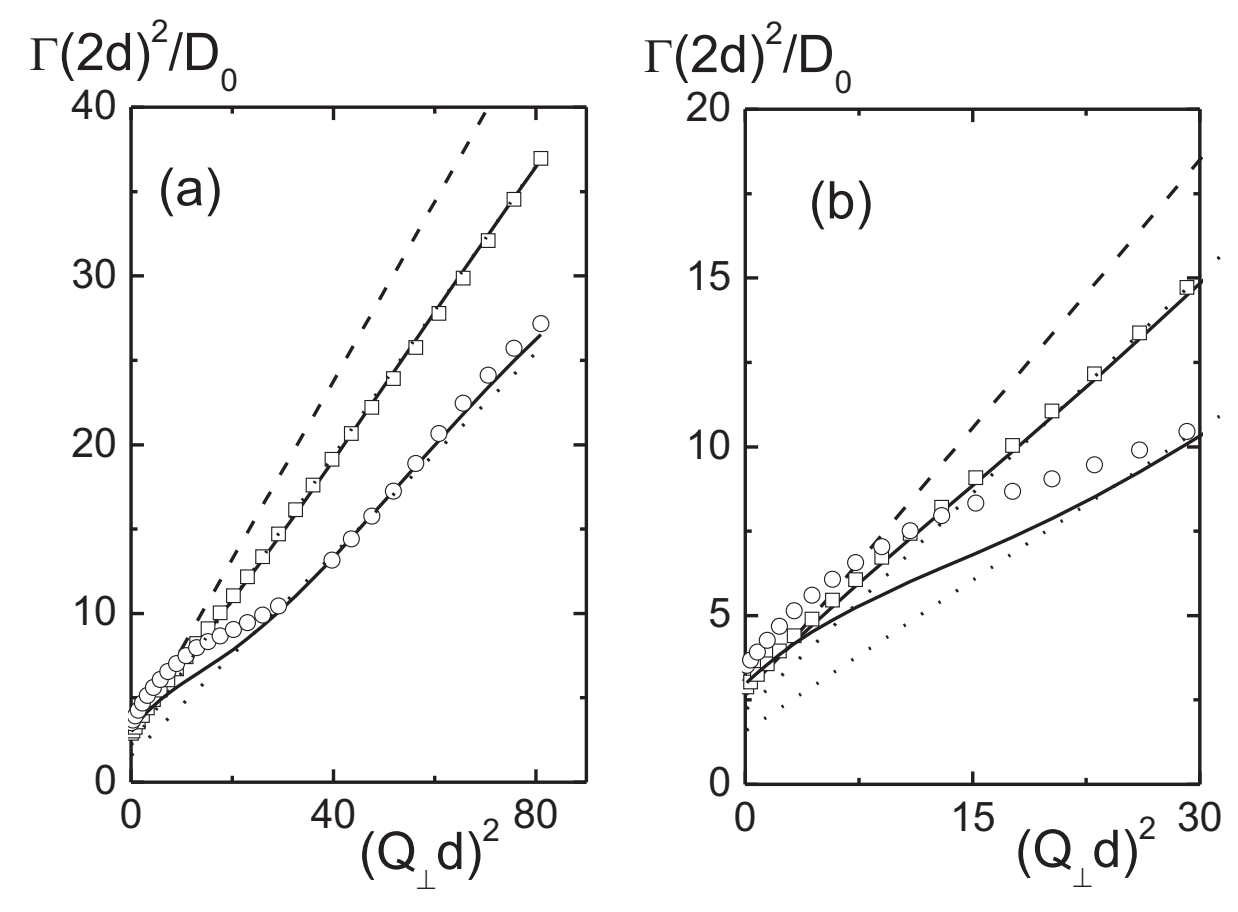

Figure 5: The initial slope as a function of the perpendicular wave-vector component for $q_{\|}=$ 1.64, and $\kappa d=0.96$. Figure (b) is a blow up of figure (a) in order to emphasize the small-wave vector region. The dashed line is for infinite dilution, the middle set of lines is for a volume fraction of $\phi=0.10$ and the lower set of curves for 0.25 . The solid lines are numerical results from the virial expansion, the data points are from simulations, and the dotted lines are the self-diffusion contributions. 
As follows from lubrication theory for a single particle, the component $D_{\perp}$, vertical to hard wall, vanishes when $z \rightarrow a$ as (see Ref.[13]),

$$
\frac{D_{\perp}(z)}{D_{0}}=\epsilon+o(\epsilon)
$$

where $\epsilon=z / a-1$. Corrections are of the order $\epsilon^{2} \ln \epsilon$. Now taking into account that for $\kappa \rightarrow+\infty$ (see Ref.[35]),

$$
\int_{a}^{+\infty} d z e^{-\kappa z} f(z) \sim e^{-\kappa a}\left[\kappa^{-1} f(a)+\kappa^{-2} f^{\prime}(a)+\cdots\right]
$$

one can show that,

$$
G_{\perp, \|}^{(1)}(\kappa d)=2(\kappa d)^{-1}+o\left((\kappa d)^{-1}\right)
$$

Similar behaviour, but with a different coefficient in front of $\kappa^{-1}$, is expected for two- and more body contributions to the vertical component for self diffusion near a wall. The asymptotic behaviour $\sim 2 /(\kappa d)$ of the single-particle perpendicular self diffusion coefficient is given by the dotted line in Fig.3. The convergence to the asymptotic values $2 /(\kappa d)$ is very slow, due to the logarithmic corrections to eq.(81). The interaction contribution to the perpendicular self diffusion coefficient varies likewise but with an as yet unknown coefficient. The lubrication contributions to the parallel components of the self diffusion coefficients have a much more complicated form as compared to the perpendicular parts in eq.(80). Hence the asymptotic formula for large $\kappa$ is not so simple as in eq.(81).

The initial slope $\Gamma$ of the EWDLS-correlation function is plotted in Figs.4 and 5 a function of $q_{\|}$and $q_{\perp}$ respectively, for the two volume fractions 0.10 and 0.25 . The data points are from the simulations, and the solid lines refer to the virial expansion (the middle curves are for $\phi=0.10$ and the lower curves for 0.25 ). The dashed line is the initial slope at infinite dilution, which can be calculated also from Brenner's hydrodynamic friction functions [4]. The figures on the right are blow-ups for small wave vectors in order to emphasize the oscillatory behaviour. The dotted lines are the curves extrapolated from very high $q$ 's, that is, the corresponding self contributions. The leading order virial expansion is surprisingly accurate up to quite high concentration. It should be noted, however, that the oscillatory behaviour at smaller wave vectors, shown in Figs.4b and 5b, is not captured by the virial expansion. These are effects from higher order interactions. The differences will be larger when the overall wave vector is smaller. In the plots in Figs.4,5, the respective wave vector components $q_{\perp} d=2.94$ and $q_{\|} d=1.64$ are fixed. For smaller values of these fixed values for the wave vectors, the oscillatory behaviour will be more pronounced and the deviation from the virial expansion is more severe. The situation is similar to the bulk initial slope, where the agreement of the self diffusive part at higher concentrations with a leading order virial expansion is better as compared to the collective $q$-dependent part. The experiments in Ref.[12] reveal that the effect of the wall diminishes at very high concentration. The interpretation of this result could be that particles very close to the wall screen the wall-hydrodynamic interactions of particles further away from 
the wall. On the other hand, however, the wall structure factor will be quite pronounced for these high concentrations, so that the diminished effect on the initial slope hints to an equally pronounced hydrodynamic function. The behaviour of the evanescent wave intensity autocorrelation function at high concentrations, beyond the validity of the virial expansion, is still unexplored.

\section{Summary and Concluding Remarks}

The interpretation of dynamic light scattering data for evanescent wave scattering is quite more complicated as compared to bulk scattering. The reason for this is that structure develops near the wall and that hydrodynamic interactions mediated through the wall are important. As a first step towards the understanding of evanescent wave scattering, we considered the initial slope of intensity auto-correlation functions. An expression for the wall-analogue of the short-time, $q$ dependent collective diffusion coefficient is derived. This formal expression is valid for arbitrary concentrations of spherical colloids. The formal expression is written in an integral form that is tractable for numerical evaluation, and explicit expressions for the various contributions to the initial slope are derived within a leading order virial expansion in concentration. In addition, a computer code has been developed with which the initial slope can be calculated for arbitrary concentration. Although numerical results given in this study are for hard-core interactions, the entire formalism is easily extended to include other types of interactions, both between the colloidal particles and between the colloids and the wall.

The main difference between the initial decay of the correlation function for very dilute systems and systems where interactions play a role is that "off-diagonal contributions" contribute, which are not simply proportional to $q_{\perp}^{2}$ and $q_{\|}^{2}$. Even within a leading order virial expansion, these "off-diagonal contributions" can be as important as the "diagonal contributions" due to inter-colloidal interactions. An additional feature of interactions is that structure builds up near the wall that affects near-wall dynamics. The corresponding wall structure factor does not asymptote to unity for large wave vectors like the bulk structure factor, which is an important feature for a quantitative interpretation of correlation functions. The wall structure factor and the hydrodynamic function are oscillating functions of the wave vector, similar to bulk systems. An distinction can be made between self-diffusive and distinct contributions to the wall diffusion coefficient. The first virial coefficient for the self part decreases monotonically with decreasing penetration depth, and thus a weaker concentration dependence is predicted as compared to the short-time bulk self diffusion coefficient. Especially for relatively large wave vectors, a good agreement between predictions for the initial slope based on the leading virial expansion and simulations that are valid for arbitrary concentrations is found. The agreement is semi-quantitative at least up to a volume fraction of 0.25 . The concentration-range where the virial expansion is a good approximation, however, becomes smaller for smaller wave vectors, similar to bulk systems.

Preliminary experiments indicate that there is good agreement with the virial expansion 
up to volume fractions of 0.25 (for the same wave-vector range for which numerical results are given in section 6) [36]. A more extended experimental test of our theory, and experiments and simulations at very high concentrations, will be published in a separate paper. An understanding of the entire time dependence of the intensity auto-correlation functions also requires further experiments and simulations. In particular, evanescent wave correlation functions exhibit a very slow decay, even for dilute systems [36], the origin of which is not yet understood. Rod-like colloids have the additional complication that orientational order exists near the wall. Virtually nothing is known yet about the dynamics of rod-like colloids near a wall, even for very dilute systems where rod-rod interactions can be neglected. These will be topics for future work.

\section{Acknowledgements}

JB would like to acknowledge financial support from NSF grants No. CBET-0348175 and CBET-0931504. JKGD and PL acknowledge financial support by the EU-FP7 Network "NanoDirect" (contract number CP-FP-213948-2).

\section{References}

\section{[1] XXXXXXXXXXXXX}

[2] P. Holmqvist, J.K.G. Dhont, P.R. Lang, hys. Rev. E, 74, 021402 (2006).

[3] P. Holmqvist, J.K.G. Dhont, P.R. Lang, J. Phys. Chem.126, 044707 (2007).

[4] H. Brenner, Chem. Eng. Sci. 16, 242 (1961).

[5] A.J. Goldman, R.G. Cox, H. Brenner, Chem. Eng. Sci. 22, 637 (1967).

[6] M. Hosoda, K. Sakai, K. Tagaki, Phys. Rev. E, 58, 6275 (1998).

[7] B. Lin, J. Yu, and S.A. Rice, Phys. Rev. E, 62, 3909 (2000).

[8] K.D. Kihm, A. Banerjee, C.K. Choi, T. Tagaki, Exp. Fluids, 37, 8011 (2004).

[9] A. Banerjee and K.D. Kihm, Phys. Rev. E, 72, 042101 (2005).

[10] For an recent overview see R. Sigel, Current Oppinion in Colloid and Interface Science, 14, 426 (2009).

[11] K.H. Lan, N. Ostrowski, D. Sornette, Phys. Rev. Lett. 57, 17 (1986).

[12] V.N. Michailidou, G. Petekidis, J.W. Swan, J.F. Brady, Phys. Rev. Lett. 102, 068302 (2009). 
[13] B.Cichocki, R.B.Jones, Ramzi Kutteh and E.Wajnryb, J.Chem.Phys. 112, 2548 (2000).

\section{[14] XXXXXXXXXXXX}

[15] A.P. Philipse, A. Vrij, J. Chem. Phys, 88, 6459 (1988).

[16] A.J. Banchio, J. Gapinski, A. Patkowski, W. Häussler, A. Fluerasu, S. Saccana, P. Holmqvist, G. Meier, M.P. Lettinga, G. Naegele, Phys. Rev. Lett. 96, 138303 (2006).

[17] A.J. Banchio, G. Naegele, J. Chem. Phys. 128, 104903 (2008).

[18] J. Gapinski, A. Patkowski, A.J. Banchio, J. Buitenhuis, P. Holmqvist, M.P. Lettinga, G. Meier, G. Naegele, J. Chem. Phys. 130, 084503 (2009).

[19] H.A. Lorentz, Versl. Kon. Akad. Wetensch. Amsterdam 5, 168 (1896).

[20] S. Kim, S.J. Karrila, Microhydrodynamics: Principles and Selected Applications, Butterworth-Heinemann, Boston, 1991.

[21] M. Abramowitz and I. A. Stegun, Handbook of Mathematical Functions, Dover, New York, 1965.

[22] P. Szymczak and B. Cichocki, J.Chem.Phys. 121, 3329 (2004).

[23] J.P. Hansen and I.R. McDonald, Theory of Simple Liquids, Academic, New York, 1976.

[24] B.Cichocki and R.B.Jones, Physica A 258, 273 (1998).

[25] J.R. Blake, Proc. Cambridge. Phil. Soc. 70, 303 (1971).

[26] L. Durlofsky, J. F. Brady and G. Bossis, J. Fluid Mech. 180, 21 (1987).

[27] B. Cichocki, M. L. Ekiel-Jeżewska and E. Wajnryb, J. Chem. Phys. 111, 3265 (1999).

[28] J. Blawzdziewicz, E. Wajnryb, Phys. Fluids. 20, 093303 (2008).

[29] S. Bhattacharya, J. Blawzdziewicz, E. Wajnryb, J. Fluid Mech. 541, 263 (2005).

[30] S. Bhattacharya, J. Blawzdziewicz, E. Wajnryb, Physica A, 356, 294, (2005).

[31] S. Bhattacharya, J. Blawzdziewicz, E. Wajnryb, J. Comput. Phys., 212, 718 (2006).

[32] M. Baron, J. Blawzdziewicz, E. Wajnryb, Phys. Rev. Lett. 100, 174502 (2008).

[33] G.K. Batchelor, J. Fluid Mech. 74, 1 (1976).

[34] B. Cichocki, B.U. Felderhof, J. Chem. Phys. 89, 1049 (1988).

[35] A. Erdèlyi, Asymptotic expansions, Dover, New York, 1987.

[36] P. Lang, unpublished experimental data. 